\title{
Une aide utile - et qui le restera longtemps
}

\section{Peter Tschudi}

Spécialiste en médecine générale FMH, président de la Commission suisse pour le développement professionnel et la qualite $(\mathrm{B} \& \mathrm{Q})$

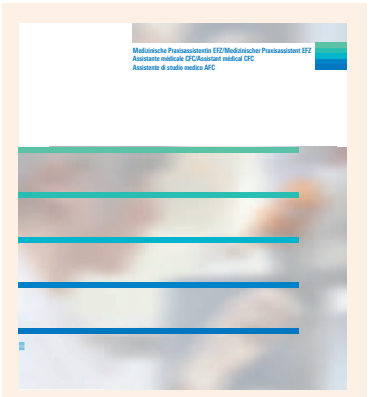

Georges Marty Lehrmittel Leitziel 1.4

Medizinische Praxisassistentin MPA

(Support de cours objectif général 1.4, Assistante médicale AM - pas encore disponible en français)

L'objectif général 1.4 complet en 3 classeurs Zurich: Careum; 2011 1000 pages en trois classeurs. $390 \mathrm{CHF}$ Produit $n^{\circ} \mathrm{C}-9231$
Correspondance: Dr Peter Tschudi Bahnhofstrasse 11 CH-8952 Schlieren
Depuis 2010, les assistantes médicales/assistants médicaux CFC (AM) sont formé-e-s selon la nouvelle ordonnance sur la formation (OrFo) et le nouveau plan de formation. Dans le cadre du processus de réforme commencé en 2006, les membres de la commission de réforme ont tenu à adapter l'ambitieuse formation d'AM aux nouvelles exigences. Le cursus a été restructuré d'après les objectifs généraux; les détails sont indiqués dans le plan de formation et l'ordonnance sur la formation (www.fmh.ch).

\section{Un support adapté au nouveau plan de formation}

Pour l'objectif général bases médicales, il existe depuis 2011 un outil extrêmement utile, publié par les Éditions Careum: le «Support de cours assistante médicale/assistant médical CFC, objectif général 1.4». Après avoir de nombreuses années exercé comme médecin généraliste et acquis une grande expérience en tant que professeur spécialisé en pathologie à l'École professionnelle d'Argovie, le Dr Georges Marty, d'Arlesheim, a adapté au nouveau plan de formation des notes très complètes dont il a fait un support de cours de grande qualité.

Réparti sur trois classeurs, le programme complet est résumé en neuf cahiers thématiques. Un cahier synoptique contient un bref résumé de chaque thème permettant aux personnes en formation de s'informer rapidement sur l'emplacement de tel ou tel contenu. Il s'y trouve aussi - de même qu'à chaque chapitre un glossaire contenant la terminologie technique essentielle ainsi qu'un index alphabétique.

\section{Un manuel complet, construit de façon didactique}

Tous les chapitres du support sont construits de façon didactique. Après une explication des notions fondamentales, ils contiennent des exercices permettant aux AM en formation de tester leur compréhension. Anatomie et physiologie sont étroitement liées. Ainsi, les bases acquièrent un sens fonctionnel et l'apprentissage s'en trouve facilité. Les maladies correspondantes sont abordées immédiatement après l'anatomie et la physiologie d'un système organique et des aspects importants de la pharmacologie sont intégrés. Cette structure didactique facilite l'appréhension des processus pathologiques. Chaque chapitre est précédé par des objectifs d'apprentissage respectant la nouvelle OrFo et préparant ainsi aux examens. Un grand nombre d'illustrations, tableaux et graphiques présentent les contenus pédagogiques avec clarté.

Ayant exercé comme professeur spécialisé en pathologie dans une école professionnelle de Zurich, ainsi que de nombreuses années dans mon propre cabinet et dans la formation d'apprentis, je sais combien il est difficile de transmettre des tableaux cliniques de façon claire et compréhensible. La compréhension de la pathologie - ou, comme elle s'appelle à présent, des bases médicales - constitue pour tout(e) AM le fondement d'un exercice satisfaisant et efficace de son métier. Nous, médecins, avons besoin qu'elles/ ils distinguent avec rapidité et compétence ce qui est important de ce qui l'est moins et priorisent de façon adéquate.

Le support de cours complet à présent disponible représente durant la formation, mais aussi plus tard comme ouvrage de référence dans chaque cabinet et pour chaque AM, un outil utile - et qui le restera. Il offre de plus au corps enseignant une aide opportune pour la préparation des cours. Je ne peux que le recommander à tous mes collègues et espère qu'il sera largement utilisé dans les écoles sur l'ensemble du territoire suisse.

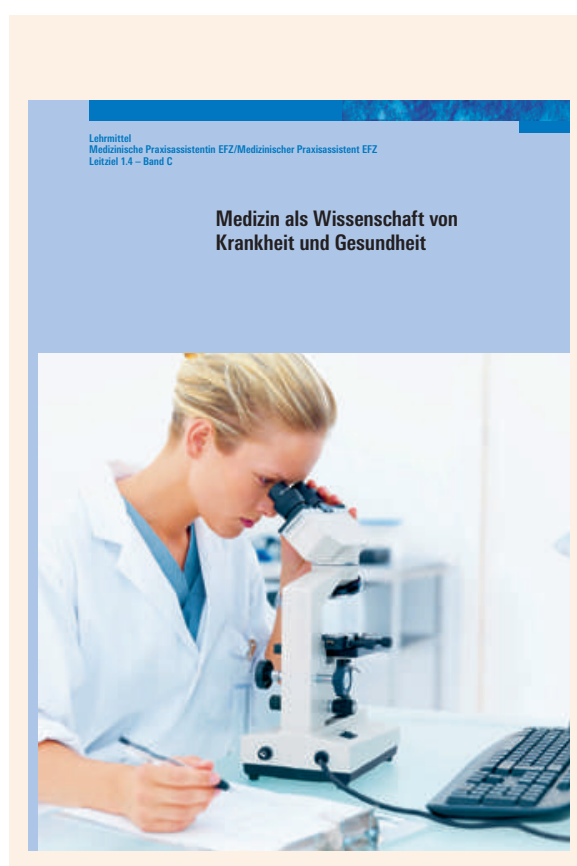

Page de couverture d'un cahier thématique. 